\title{
On the Similarity Based Impulsive Noise Removal Technique for Multichannel Images
}

\author{
Bogdan Smolka \\ Department of Automatic Control, \\ Silesian University of Technology, \\ Akademicka 16, 44-101, Gliwice, Poland
}

\begin{abstract}
In this paper a novel approach to the problem of impulsive noise reduction in color images based on the nonparametric density estimation is presented. The basic idea behind the new image filtering technique is the maximization of the similarities between pixels in a predefined filtering window. The new method is faster than the standard vector median filter and preserves better edges and fine image details.
\end{abstract}

\section{Introduction}

A number of nonlinear, multichannel filters, which utilize correlation among multivariate vectors using various distance measures, have been proposed [1-11]. The most popular nonlinear, multichannel filters are based on the ordering of vectors in a predefined moving window. The output of these filters is defined as the lowest ranked vector according to a specific vector ordering technique.

Let $\mathbf{F}(x)$ represents a multichannel image and let $W$ be a window of finite size $n+1$ (filter length). The noisy image vectors inside the filtering window $W$ are denoted as $\mathbf{F}_{j}, j=0,1, \ldots, n$. If the distance between two vectors $\mathbf{F}_{i}, \mathbf{F}_{j}$ is denoted as $\rho\left(\mathbf{F}_{i}, \mathbf{F}_{j}\right)$ then the scalar quantity $R_{i}=\sum_{j=0}^{n} \rho\left(\mathbf{F}_{i}, \mathbf{F}_{j}\right)$ is the distance associated with the noisy vector $\mathbf{F}_{i}$. The ordering of the $R_{i}$ 's: $R_{(0)} \leq$ $R_{(1)} \leq \ldots \leq R_{(n)}$, implies the same ordering to the corresponding vectors $\mathbf{F}_{i}$ : $\mathbf{F}_{(0)} \leq \mathbf{F}_{(1)} \leq \ldots \leq \mathbf{F}_{(n)}$. Nonlinear ranked type multichannel estimators define the vector $\mathbf{F}_{(0)}$ as the filter output. However, the concept of input ordering, initially applied to scalar quantities is not easily extended to multichannel data, since there is no universal way to define ordering in vector spaces. To overcome this problem, distance functions are often utilized to order vectors, [1,2].

All standard filters detect and replace well noisy pixels, but their property of preserving pixels which were not corrupted by the noise process is far from the ideal. In this paper the construction of a simple, efficient and fast filter, which removes noisy pixels, but has the ability of preserving original image pixel values, is presented.

\section{Proposed Algorithm}

\subsection{Gray-Scale Images}

Let us assume a filtering window $W$ containing $n+1$ image pixels, $\left\{F_{0}, F_{1}, \ldots, F_{n}\right\}$, where $n$ is the number of neighbors of the central pixel $F_{0}$, and let us define the 
similarity function $\mu:[0 ; \infty) \rightarrow \mathbf{R}$ which is non-ascending and convex in $[0 ; \infty)$ and satisfies $\mu(0)=1, \mu(\infty)=0$. The similarity between two pixels of the same intensity should be 1 , and the similarity between pixels with minimal and maximal gray scale values should be very close to 0 . The function $\mu\left(F_{i}, F_{j}\right)$ defined as $\mu\left(F_{i}, F_{j}\right)=\mu\left(\left|F_{i}-F_{j}\right|\right)$ satisfies the three required conditions.

Let us additionally define the cumulated sum $M$ of similarities between a given pixel and all other pixels belonging to window $W$. For the central pixel let us introduce $M_{0}$ and for the neighbors of $F_{0}$ let us define $M_{k}$ as

$$
M_{0}=\sum_{j=1}^{n} \mu\left(F_{0}, F_{j}\right), \quad M_{k}=\sum_{\substack{j=1 \\ j \neq k}}^{n} \mu\left(F_{k}, F_{j}\right),
$$

which means that for $F_{k}$ which are neighbors of $F_{0}$ we do not take into account the similarity between $F_{k}$ and $F_{0}$, which is the main idea behind the new algorithm. The omission of the similarity $\mu\left(F_{k}, F_{0}\right)$ when calculating $M_{k}$, privileges the central pixel, as in the calculation of $M_{0}$ we have $n$ similarities $\mu\left(F_{0}, F_{k}\right)$, $k=1,2, \ldots, n$ and for $M_{k}, k>0$ we have only $n-1$ similarity values, as the central pixel $F_{0}$ is excluded from the calculation of the sum $M_{k}$.

In the construction of the new filter, the reference pixel $F_{0}$ in the window $W$ is replaced by one of its neighbors if $M_{0}<M_{k}, k=1, \ldots, n$. If this is the case, then $F_{0}$ is replaced by that $F_{k^{*}}$ for which $k^{*}=\arg \max M_{k}, k=1, \ldots, n$.

In other words $F_{0}$ is detected as being corrupted if $M_{0}<M_{k}, k=1, \ldots, n$ and is replaced by its neighbors $F_{k}$ which maximizes the sum of similarities $M$ between all pixels of $W$ excluding the central pixel. This is illustrated in Fig. 1.

The basic assumption is that a new pixel must be taken from the window $W$ (introducing pixels which do not occur in the image is prohibited like in the $\mathrm{VMF}$ ). For this purpose $\mu$ must be convex, which means that in order to find a maximum of the sum of similarity functions $M$ it is sufficient to calculate the values of $M$ only in points $F_{0}, F_{1}, \ldots, F_{n},[11]$.

\subsection{Color Images}

The presented approach can be applied in a straightforward way to color images. We use the similarity function defined by $\mu\left\{\mathbf{F}_{i}, \mathbf{F}_{j}\right\}=\mu\left(\| \mathbf{F}_{i}-\mathbf{F}_{j}\right) \|$ where $\|\cdot\|$ denotes the specific vector norm. Now in exactly the same way we can maximize the total similarity function $M$ for the vector case.

Several convex functions were applied in order to compare the new filtering scheme with the standard filters used in color image processing presented in Tab. 1 and good results were obtained, (see Tab. 2), when applying the following similarity functions, which can be treated as kernels of nonparametric estimation, $[12,13]$.

$$
\begin{gathered}
\mu_{0}(x)=\exp \left\{-\left(\frac{x}{h}\right)^{2}\right\}, \mu_{1}(x)=\exp \left\{-\frac{x}{h},\right\}, \mu_{2}(x)=\frac{1}{1+x / h}, h \in(0 ; \infty), \\
\mu_{3}(x)=\frac{1}{(1+x)^{h}}, \mu_{4}(x)=1-\frac{2}{\pi} \arctan \left(\frac{x}{h}\right), \mu_{5}(x)=\frac{2}{1+\exp \left\{\frac{x}{h}\right\}}, h \in(0 ; \infty),
\end{gathered}
$$




$$
\mu_{6}(x)=\frac{1}{1+x^{h}}, \quad \mu_{7}(x)=\left\{\begin{array}{cl}
1-x / h & \text { if } x \leq h, \\
0 & \text { if } x>h,
\end{array} \quad h \in(0 ; \infty) .\right.
$$

It is interesting to note, that the best results were achieved for the simplest similarity function $\mu_{7}(x)$, which allows to construct a fast noise reduction algorithm. In the multichannel case, we have

$$
M_{0}=\sum_{j=1}^{n} \mu\left(\mathbf{F}_{0}, \mathbf{F}_{j}\right), \quad M_{k}=\sum_{\substack{j=1 \\ j \neq k}}^{n} \mu\left(\mathbf{F}_{k}, \mathbf{F}_{j}\right)
$$

where $\left.\rho\left\{\mathbf{F}_{i}, \mathbf{F}_{k}\right\}=\| \mathbf{F}_{k}-\mathbf{F}_{l}\right) \|$ and $\|\cdot\|$ is the $L_{2}$ vector norm, as it yields the best results.

Applying the linear similarity function $\mu_{7}$ we obtain

$$
\mu\left(\mathbf{F}_{i}, \mathbf{F}_{k}\right)=\left\{\begin{array}{cc}
1-\frac{\rho\left(\mathbf{F}_{i}, \mathbf{F}_{k}\right)}{h} & \text { for } \rho\left(\mathbf{F}_{i}, \mathbf{F}_{k}\right)<h \\
0 & \text { otherwise }
\end{array}\right.
$$

Then we have from (2)

$$
M_{0}=n-\frac{1}{h} \sum_{j=1}^{n} \rho\left(\mathbf{F}_{0}, \mathbf{F}_{j}\right) \quad M_{k}=\sum_{\substack{j=1, j \neq k}}^{n}\left(1-\frac{\rho\left(\mathbf{F}_{k}, \mathbf{F}_{j}\right)}{h}\right)=n-1-\frac{1}{h} \sum_{j=1}^{n} \rho\left(\mathbf{F}_{k}, \mathbf{F}_{j}\right)
$$

In this way the difference between $M_{0}$ and $M_{k}$ is

$$
\begin{gathered}
M_{0}-M_{k}=n-\frac{1}{h} \sum_{j=1}^{n} \rho\left(\mathbf{F}_{0}, \mathbf{F}_{j}\right)-\left[n-1-\frac{1}{h} \sum_{j=1}^{n} \rho\left(\mathbf{F}_{k}, \mathbf{F}_{j}\right)\right]= \\
=1-\frac{1}{h} \sum_{j=1}^{n}\left[\rho\left(\mathbf{F}_{0}, \mathbf{F}_{j}\right)-\rho\left(\mathbf{F}_{k}, \mathbf{F}_{j}\right)\right], \\
M_{0}-M_{k}>0 \text { if } h>\sum_{j=1}^{n}\left[\rho\left(\mathbf{F}_{0}, \mathbf{F}_{j}\right)-\rho\left(\mathbf{F}_{k}, \mathbf{F}_{j}\right)\right] .
\end{gathered}
$$

If this condition is satisfied, then the central pixel is considered as not disturbed by the noise process, otherwise the pixel $\mathbf{F}_{k}$ for which the cumulative similarity value achieves maximum, replaces the central noisy pixel. In this way the filter changes the central pixel only when it is really noisy and preserves the original undistorted image structures, (Figs. 1 and 2).

It is easy to observe that the construction of the new filter is quite similar to the standard VMF. Instead of the function $R_{k}$ used in the VMF, a modified cumulative distance function $R_{0}$ is proposed

$$
R_{k}=\left\{\begin{array}{cl}
-h+\sum_{j=1}^{n} \rho\left(\mathbf{F}_{k}, \mathbf{F}_{j}\right), & \text { for } \quad k=0, \\
\sum_{j=1}^{n} \rho\left(\mathbf{F}_{k}, \mathbf{F}_{j}\right), & \text { for } \quad k=1, \ldots, n
\end{array}\right.
$$


and in the same way as in VMF, the original vector $\mathbf{F}_{0}$ in the filter window $W$ is being replaced by $\mathbf{F}_{k^{*}}$ such that $k^{*}=\arg \min _{k} R_{k}$.

It is easy to notice that the above construction is equivalent to the condition expressed in (6). Now, instead of maximizing the cumulative similarity $M_{k}$, we minimize the modified cumulative distance $R_{k}$. In this way, the condition for retaining the original image pixel is: $R_{0} \leq R_{k}, k=1, \ldots, n$, which leads to the condition

$$
\begin{gathered}
-h+\sum_{j=1}^{n} \rho\left(\mathbf{F}_{0}, \mathbf{F}_{j}\right) \leq \sum_{j=1}^{n} \rho\left(\mathbf{F}_{k}, \mathbf{F}_{j}\right), \quad k=1, \ldots, n, \\
R_{0} \leq R_{k} \text { if } \quad h \geq \sum_{j=1}^{n}\left[\rho\left(\mathbf{F}_{0}, \mathbf{F}_{j}\right)-\rho\left(\mathbf{F}_{k}, \mathbf{F}_{j}\right)\right], k=1, \ldots, n .
\end{gathered}
$$

The construction of the new filter is very similar to that of VMF, however the major difference is the omitting of the central pixel $\mathbf{F}_{0}$, when calculating $R_{k}, k>0$. This scheme, based on the simple leave-one-out scheme, is the most important feature of the new algorithm. As the central pixel is suspected to be noisy, it is not taken into consideration, when calculating the distances associated with the neighbors of $\mathbf{F}_{0}$. In this way the filter replaces the central pixel only when it is really noisy, while preserving the original undisturbed image structures.

As widely known, VMF has the disadvantage of replacing too many uncorrupted image pixels. It is improved in the new filter design by setting positive $h$ values, which forces the filter to preserve uncorrupted, original pixels, but still enables to remove corrupted ones. Moreover, $h$ can be controlled for the best effectiveness of the filter depending on image structure and noise statistics.

It is easy to observe that the presented method is faster than VMF. It can be shown using a simple matrix representation, (for the sake of simplicity in the 4-neighborhood system case). In order to find $R_{k^{*}}$ using the VMF method we have to add the elements in rows or columns of the symmetric matrix $\mathbf{T}_{V M F}$

$$
\mathbf{T}_{V M F}=\left[\begin{array}{ccccc}
0 & \rho_{01} & \rho_{02} & \rho_{03} & \rho_{04} \\
\rho_{10} & 0 & \rho_{12} & \rho_{13} & \rho_{14} \\
\rho_{20} & \rho_{21} & 0 & \rho_{23} & \rho_{24} \\
\rho_{30} & \rho_{31} & \rho_{32} & 0 & \rho_{34} \\
\rho_{40} & \rho_{41} & \rho_{42} & \rho_{43} & 0
\end{array}\right], \quad \mathbf{T}_{N E W}=\left[\begin{array}{ccccc}
-h & \rho_{01} & \rho_{02} & \rho_{03} & \rho_{04} \\
0 & 0 & \rho_{12} & \rho_{13} & \rho_{14} \\
0 & \rho_{21} & 0 & \rho_{23} & \rho_{24} \\
0 & \rho_{31} & \rho_{32} & 0 & \rho_{34} \\
0 & \rho_{41} & \rho_{42} & \rho_{43} & 0
\end{array}\right]
$$

where $\rho_{i j}=\rho_{j i}=\rho\left(\mathbf{F}_{i}, \mathbf{F}_{j}\right)$. Obviously, the symmetry of the matrix $\mathbf{T}_{V M F}$ causes that effectively we have to compute 10 distances (36 in the 8-neighborhood case) and then to make 15 additions (63 in the 8-neighborhood case). For the implementation of the new filter (matrix $\mathbf{T}_{N E W}$ ), the number of distances we need, is still 10 but there is only 11 additions ( 55 in the 8-neighborhood case), so the new filter is faster than VMF. 


\section{Results}

For evaluation purposes, the color test image $L E N A$ was corrupted with impulsive noise defined by $\mathbf{x}_{i j}=\mathbf{v}_{i j}$ with probability $p$, where $i, j$ define a pixel position, $p$ describes the intensity of the noise process, $\mathbf{x}_{i j}$ denotes the original image pixel and $\mathbf{v}_{i j}$ denotes a pixel corrupted by the noise process $\mathbf{v}_{i j}=\left\{\nu_{R}, \nu_{G}, \nu_{B}\right\}$, where $\nu_{R}, \nu_{G}, \nu_{B}$ are random integer variables from the interval $[0,255]$ updated for each corrupted pixel.

The root of the mean squared error (RMSE), peak signal to noise ratio (PSNR), normalized mean square error (NMSE) [1,2] were used for the analysis. The comparison shows that the new filter outperforms by far the standard vector median filter, which can be treated as a reference filter, and other filters listed in Tab. 1. The efficiency of the new filtering technique is shown in Tab. 2 and in Fig. 2.

The new algorithm presented in this paper can be seen as a fast modification and improvement of the Vector Median Filter. The comparison with standard color image processing filters shows that the new filter outperforms the standard procedures used in color image processing. Another advantage of the proposed filtering class is its lower computational complexity compared to the VMF, which makes the new filter class interesting for real-time applications

\section{Conclusions}

The new algorithm presented in this paper can be seen as a fast modification and improvement of the Vector Median Filter. The comparison with standard color image processing filters, (Tab. 2, Fig. 2) shows that the new filter outperforms the standard procedures used in color image processing, when the impulse noise is to be eliminated.

\begin{tabular}{|c|c|}
\hline Notation & Filter \\
\hline AMF & Arithmetic Mean Filter \\
\hline VMF & Vector Median Filter \\
\hline BVDF & Basic Vector Directional Filter \\
\hline HDF & Hybrid Directional Filter \\
\hline AHDF & Adapt. Hybrid Directional Filter \\
\hline DDF & Directional-Distance Filter \\
\hline
\end{tabular}

Table 1. Filters taken for comparison with the proposed adaptive filter, [1,2].

\section{References}

1. I. Pitas, A. N. Venetsanopoulos, 'Nonlinear Digital Filters : Principles and Applications', Kluwer Academic Publishers, Boston, MA, (1990)

2. K.N. Plataniotis, A.N. Venetsanopoulos, 'Color Image Processing and Applications', Springer Verlag, (June 2000)

3. I. Pitas, P. Tsakalides, Multivariate ordering in color image processing, IEEE Trans. on Circuits and Systems for Video Technology, 1, 3, 247-256, (1991) 
4. D.G. Karakos, P.E. Trahanias, Combining vector median and vector directional filters: The directional - distance filters, Proceedings of the IEEE Conf. on Image Processing, ICIP-95, 171-174, (October 1995)

5. I. Pitas, A.N. Venetsanopoulos, Order statistics in digital image processing, Proceedings of IEEE, 80, 12, 1893-1923, (1992)

6. J. Astola, P. Haavisto, Y. Neuovo, Vector median filters, IEEE Proceedings, 78, 678-689, (1990)

7. P.E. Trahanias, A.N. Venetsanopoulos, Vector directional filters: A new class of multichannel image processing filters, IEEE Trans. on Image Processing, 2, 4, 528534, (1993)

8. M. Gabbouj, F.A. Cheickh, Vector Median-Vector Directional hybrid filter for color image restoration, Proceedings of EUSIPCO-96, 879-881, (1996)

9. K.N. Plataniotis, D. Androutsos, A.N. Venetsanopoulos, Colour Image Processing Using Fuzzy Vector Directional Filters, Proceedings of the IEEE Workshop on Nonlinear Signal/Image Processing, Greece, 535-538, (1995)

10. K.N. Plataniotis, D. Androutsos, V. Sri, A.N. Venetsanopoulos, A nearest neighbour multichannel filter, Electronic Letters, 1910-1911, (1995)

11. B. Smolka, A. Chydzinski, K. Wojciechowski, K. Plataniotis, A.N. Venetsanopoulos, On the reduction of impulsive noise in multichannel image processing, Optical Engineering, 40, 6, 902-908, (2001)

12. B.W. Silverman, "Density Estimation for Statistics and Data Analysis", London, Chapman and Hall, (1986)

13. D.W. Scott, "Multivariate Density Estimation", New York, John Wiley, (1992)

\begin{tabular}{|c|c|c|c|}
\hline METHOD & NMSE $\left[10^{-4}\right]$ & RMSE & PSNR [dB] \\
\hline NONE & 514.95 & 32.165 & 17.983 \\
\hline AMF & 82.863 & 12.903 & 25.917 \\
\hline VMF & 23.304 & 6.842 & 30.149 \\
\hline BVDF & 29,074 & 7.643 & 30.466 \\
\hline HDF & 22.845 & 6.775 & 31.513 \\
\hline AHDF & 22,603 & 6,739 & 31.559 \\
\hline DDF & 24.003 & 6.944 & 31,288 \\
\hline \multicolumn{4}{|c|}{ FILTERING KERNELS } \\
\hline$\mu_{0}(x)$ & 5.056 & 3.163 & 38.137 \\
\hline$\mu_{1}(x)$ & 4.959 & 3.157 & 38.145 \\
\hline$\mu_{2}(x)$ & 5.398 & 3.294 & 37.776 \\
\hline$\mu_{3}(x)$ & 9.574 & 4.387 & 35.288 \\
\hline$\mu_{4}(x)$ & 5.064 & 3.190 & 38.054 \\
\hline$\mu_{5}(x)$ & 4.777 & 3.099 & 38.307 \\
\hline$\mu_{6}(x)$ & 11.024 & 4.707 & 34.675 \\
\hline$\mu_{\mathbf{7}}(\mathbf{x})$ & $\mathbf{4 . 6 9 3}$ & $\mathbf{3 . 0 7 2}$ & $\mathbf{3 8 . 3 8 4}$ \\
\hline \multicolumn{4}{|c}{}
\end{tabular}

Table 2. Comparison of the new algorithm based on different kernel functions with the standard techniques, using the LENA color image contaminated by $10 \%$ of impulsive noise. 


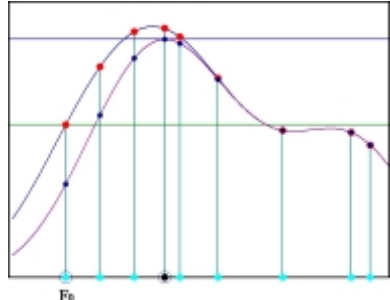

a)

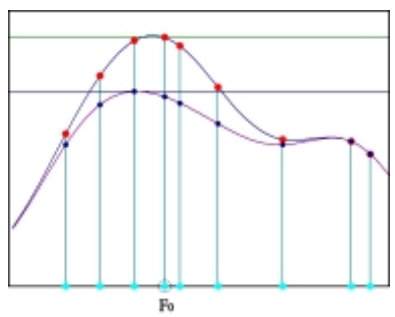

d)

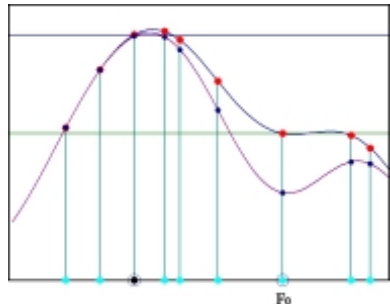

g)

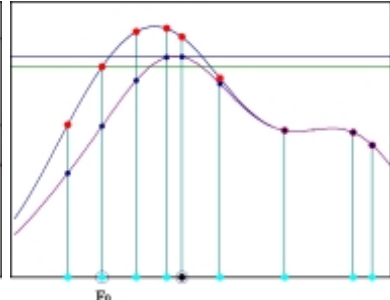

b)

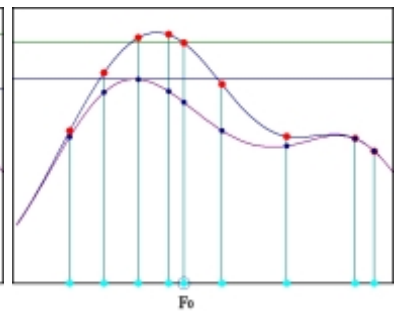

e)

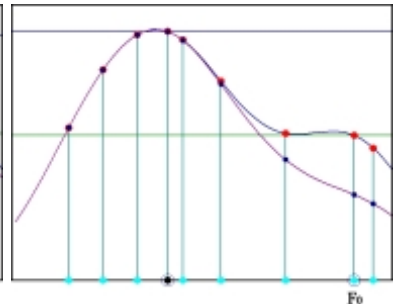

h)

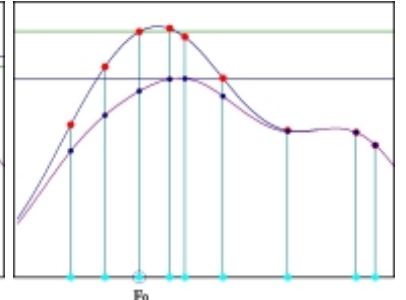

c)

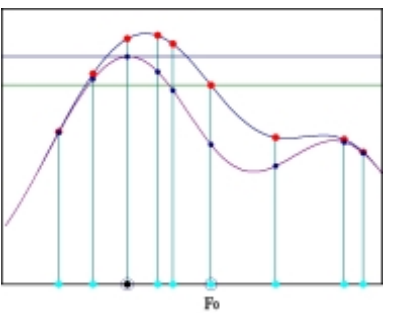

f)

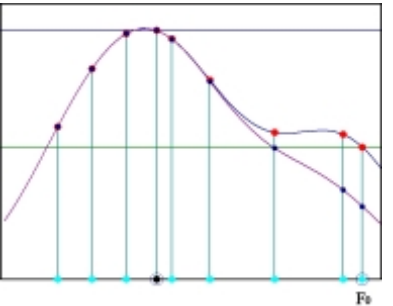

i)

Fig. 1. Illustration of the new filter construction. The supporting window $W$ of size $3 \times 3$ contains 9 pixels of intensities $\{15,24,33,41,45,55,72,90,95\}$. Each of the graphs from a) to i) shows the dependence of $M_{0}$ and $M_{/ 0},\left(M_{/ 0}<M_{0}\right)$, where $M_{/ 0}$ denotes the cumulative similarity value with rejected central pixel $F_{0}$ on the pixel gray scale value. Graph a) shows the plot of $M_{0}$ and $M_{/ 0}$ for $F_{0}=15$, plot b) for $F_{0}=24$ and so on till plot plot i) which shows the graphs of $M_{0}$ and $M_{/ 0}$ for $F_{0}=95$. The arrangement of pixels surrounding the central pixel $F_{0}$ is not relevant. The central pixel will be replaced in cases: $(\mathbf{a}),(\mathbf{b}),(\mathbf{f})-(\mathbf{i})$, as in those cases there exists a pixel $F_{i}$ for which $M_{0}<M_{i}$. 

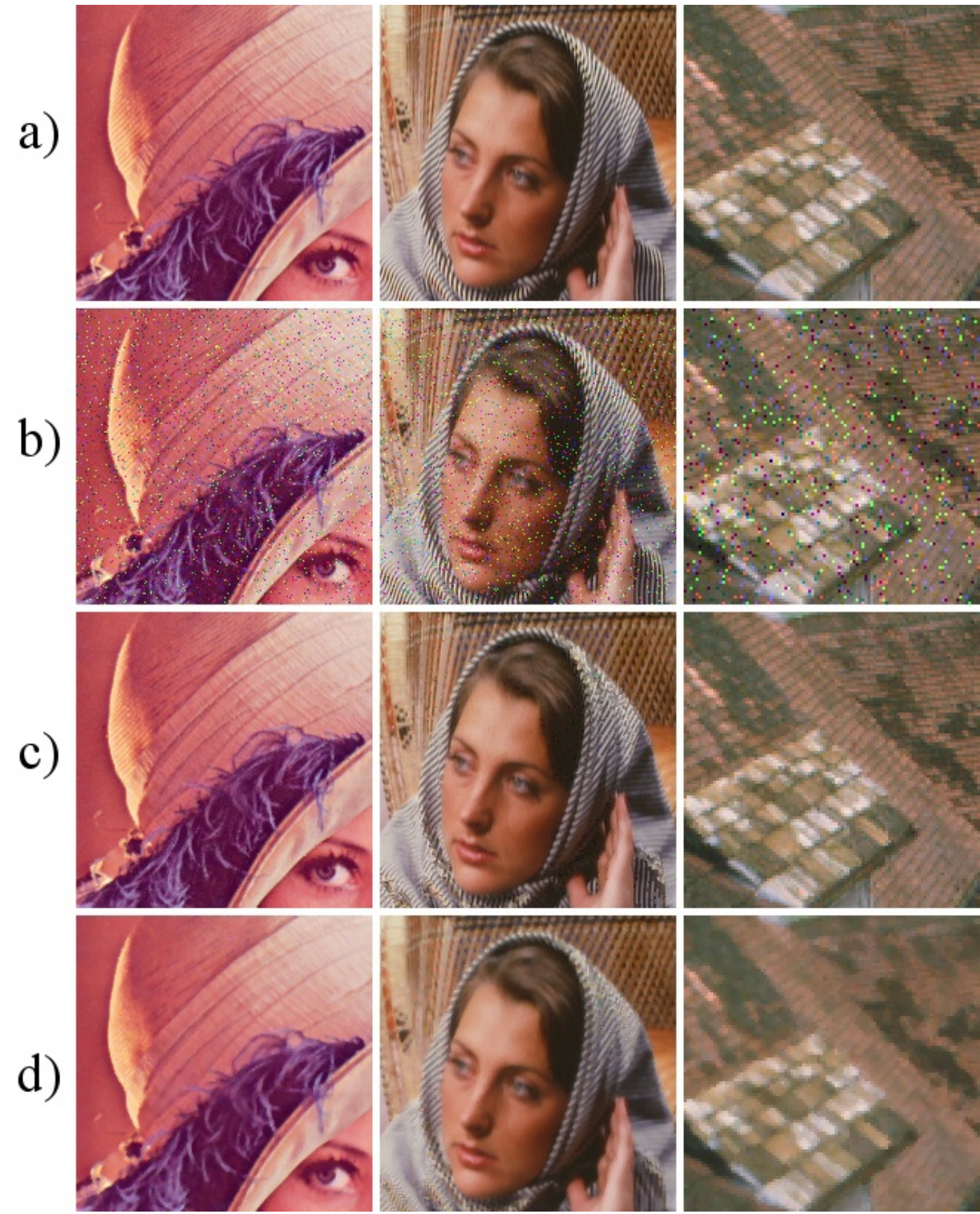

Fig. 2. Comparison of the efficiency of proposed filter with the VMF: a) parts of the $L E N A, B A R B A R A$ and GOLDHIL images, b) images contaminated by $6 \%$ impulsive noise, c) images restored using the proposed technique, $\mathbf{d}$ ) the result of the filtering with the vector median. 